Traditional Medicine and Modern Medicine

Vol. 2, No. 3 (2019) 127-133

(C) Institutes of Integrative Medicine, Fudan University and

World Century Publishing Corporation

DOI: $10.1142 /$ S2575900019500113

\title{
The Üstikuddus sherbiti in the treatment of abnormal cold and dry-type depression patients with comorbid anxiety: A randomized and controlled clinical trial
}

\author{
Aman Gul ${ }^{1,2,3}$, Nassirhadjy Memtily ${ }^{4}$, Pirdun Mijit ${ }^{5}$, Mihriban $\operatorname{Imin}^{6}$, Hua Rui ${ }^{6}$, \\ Palidan Wushuer ${ }^{6, *}, *$ and Ainiwaer Talifu ${ }^{7, \dagger, *}$ \\ ${ }^{1}$ Department of Integrative Medicine \\ Huashan Hospital, Fudan University \\ 12 Middle Urumqi Road, Shanghai 200040, P. R. China \\ ${ }^{2}$ Institute of Theories and Application, Institutes of Integrative Medicine \\ Fudan University, 12 Middle Urumqi Road \\ Shanghai 200040, P. R. China \\ ${ }^{3}$ Central Laboratory, Xinjiang Medical University \\ Urumqi, Xinjiang Uygur Autonomous Region 830011, P. R. China \\ ${ }^{4}$ Traditional Uyghur Medicine Institute \\ Xinjiang Medical University, Urumqi, Xinjiang Uygur \\ Autonomous Region 830011, P. R. China \\ ${ }^{5}$ Department of Public Health, Xinjiang Medical University \\ Urumqi, Xinjiang Uygur Autonomous Region 830011, P. R. China \\ ${ }^{6}$ Department of Psychosomatic, the Affiliated Chinese Medicine Hospital \\ Xinjiang Medical University, Urumqi Xinjiang \\ Uygur Autonomous Region 830000, P. R. China \\ ${ }^{7}$ Hospital of Xinjiang Traditional Uyghur Medicine \\ Urumqi, Xinjiang Uygur Autonomous Region 830049, P. R. China \\ *pld425@sina.com \\ †anwar717@sohu.com
}

Received 17 June 2019; Accepted 18 November 2019; Published 30 December 2019

Objective: To evaluate the clinical effect of Üstikuddus sherbiti (US), a traditional herbal prescription of Uyghur medicine, in the treatment of cold and dry-type major depression disorder (MDD) patients with comorbid anxiety.

Methods: A total of 150 cold and dry-type depressive patients with comorbid anxiety were randomly divided into three groups of 50 patients; then they were, respectively, treated with US, modern medicine Fluoxetine, and the combination of Fluoxetine and US. The depression degrees of patients were evaluated zero and six weeks after the treatment based on the Hamilton Anxiety Scale (HAMA), in the clinical study. Analysis of variance (ANOVA) was used to detect significant differences at various time points or among the three groups. Statistical analysis was performed by SPSS version 21.0.

Results: The main results are as follows: After taking the medicine, the depressive condition of patients with comorbid anxiety in the three groups was getting better with the passage of time; particularly, the clinical efficacy of

¥Corresponding authors. 
US combined with Fluoxetine was significantly improved, indicating that the effect of combined treatment is better than treatment with pure Fluoxetine or pure US treatment. Statistical difference exists between the end time point within groups, but not among the three groups. Moreover, in the detection process of the patient's physical indicators, no obvious side effects were found. These results together suggested that US can strengthen the curative effect of modern medicine in the treatment of depression, which would lay a foundation for studying the molecular mechanism and potential target of US.

Conclusion: Fluoxetine and US combination therapy played a significant role in the treatment of depressive symptoms, suggesting that it can improve the curative effect of depression. The study provided a new way of thinking to clarify the US molecular mechanism for the treatment of depression and potential target.

Keywords: Üstikuddus sherbiti (US); comorbid anxiety and depression; randomized controlled trial; HAMA.

\section{Introduction}

Major depression disorder (MDD) is a common mental suffering characterized by both psychological and physiological symptoms, including emotional depression, concentration and cognitive impairment, slow thinking, tiredness, low selfesteem, guilt, poor sleep, appetite disturbance, lack of interest, and being unconfident. ${ }^{1,2}$ According to the report by the World Health Organization (WHO), the global incidence of depression is about $8-12 \%$, and the depressed population amounts to 350 million people all over the world, $10-15 \%$ of which may eventually attempt for suicide. ${ }^{3,4}$ In China, the numbers of college students suffering with severe and mild depression, respectively, account for $14.7 \%$ and $42.1 \%$, it means that $60 \%$ of students are associated with varying degrees of depression or some other mental disorders. MDD has become the main cause of death among global college students. $^{5-7}$ It is expected that by 2020, MDD will likely become the second largest human disease only behind the heart disease. ${ }^{8-10}$ Therefore, it is urgent to intensify the prevention and treatment of depression.

Comorbidity of psychiatric syndromes is quite common, depression with anxiety is a common clinical symptom, and the prevalence of comorbid anxiety disorder and MDD is frequent and perhaps as high as more than half. ${ }^{11-13}$ Many scholars believe that both have a common pathogenic basis. ${ }^{11-13}$

In the early stages, anti-depressants, mainly including tricyclic anti-depressant (TCA) and monoamine oxidase inhibitor (MAOI), may have certain positive effects on depression, but some anti-cholinergic effects that impair the central memory are unavoidable. In 1980s, a new generation of anti-depressants such as SSRI, SNRI, NDRI, NaSSA, and SARI $^{14}$ have obtained curative effects in the treatment of depression but their disadvantages are uncontrolled dosage, slow release, adverse reactions, and expensive cost. ${ }^{15-18}$ Current clinical treatment of depression still depends on modern medicine, which, in spite of a certain positive effect, has different degrees of side effects and drug dependence. Therefore, there is high need of a novel medicine that is efficient, nontoxic, has low side effects, low adverse reactions, and drug dependences. Traditional herbal prescriptions of ethnic medicines might be one of the sources.

Traditional Uyghur medicine (TUM) is an important part of traditional Chinese medicine (TCM) particularly ${ }^{19}$; the therapy based on Humorism (body fluid) and munziq (ripening abnormal body fluid compounds) shows significant advantages and features in the prevention and treatment of depression and other complex diseases. ${ }^{19-21}$ The descriptions on etiology, pathogenesis, diagnosis, and treatment of depression are scattered through ancient medical books. In TUM, depression is called kara sewda, kara sefra, and hamushluk. ${ }^{20,22}$ The abnormal cold and dry-type body fluid (abnormal sewda) is a major cause of depression and many incurable diseases.

Üstikuddus sherbiti (US; Üstikuddus Compound, Yinao Wusitikudusi Tangjiang, Xinjiang Drug Approval No. M20041577; preparation produced in Xinjiang Traditional Uyghur Medicine Hospital) is used for the treatment and prevention of depression in TUM. ${ }^{23-25}$ US was made from 12 kinds of herbs such as Lavandula angustifolia Mill., Adiantum flabellulatum L., Paeonia lactiflora Pall., Anchusa italica Retz., Radix liquiritiae, rose petals, raisins, celery seeds, Althaea rosea L., Viola tianschanica Maxim., Cordia dichotoma seeds, and Foeniculum vulgare Mill. ${ }^{23-25}$ It is mainly used for the treatment of depression, migraine, neurasthenia, and other diseases. Clinical studies have shown that it has a significant effect on the treatment of depression, but the mechanism of drug effect still remains unknown. In clinical practice, the detailed treatment mechanism of the mixture is as follows: It is used to recuperate cold and drytype body fluid, and then mushil (abnormal cold and dry-type body fluid scavenger, remover) compels the maturing cold and dry-type body fluid to get discharged from the organism, together contributing to the equilibrium of various body's fluids and rehabilitation from multiple diseases. ${ }^{19}$ US is believed to be the best choice for preventing and curing complex diseases such as depression.

In this study, the administration of US or modern medicine Fluoxetine is found to have a good therapeutic effect on 
the depressive condition of patients with comorbid anxiety. However, the combination of US and Fluoxetine exhibited a better curative efficacy compared with the individual therapy groups, indicating that taking US can improve the clinical effect of modern medicine and promote the rehabilitation of patients. Our study will provide a novel approach with traditional medicine in the treatment of depression.

\section{Materials and Methods}

\section{Research objects}

Our research objects included a total of 150 patients from the Affiliated Hospital of Chinese Medicine, Xinjiang Medical University, who were diagnosed with major depression at the Outpatient Department of Psychotherapy during the period from July 2015 to October 2017. All volunteers were informed and signed consent was obtained from them. The Ethics Committee License No. is 2013XE-KS01.

\section{Diagnostic criteria for the modern medicine inclusion}

In this study, the patients with depression were diagnosed according to the International Classification of Diseases-10, i.e., the mental and behavior disorders diagnostic criteria, officially announced by WHO. ${ }^{26}$ Low mood is mainly manifested as follows: (1) Loss of interest, no pleasure; (2) loss of energy or fatigue; (3) psychomotor retardation or agitation; (4) low self-assessment, self-blame, or guilt; (5) lack of communication skills or conscious thinking; (6) repeated idea of dying or Dutch Act, and self-injurious behavior; (7) sleep disorders, such as insomnia, waking up early, or sleeping for a long time; (8) loss of appetite or weight; and (9) hyposexuality.

Standards of illness duration: the patients have met with the criteria and the severity of symptoms for at least two weeks.

\section{Exclusion criteria}

Exclusion of organic mental disorders or mental activity and addictive substance-induced depression.

\section{Severity criteria}

According to Hamilton Depression Scale (HAMD-24) $\geqq 21$, and the Hamilton Anxiety Scale (HAMA) $\geqq 21$, the depressive patients at 0-4 grades were divided into five levels of grading standards, which are as follows: 0 : not have, 1 : mild or moderate, 2: medium, 3: severe, and 4: more severe. Total score reflects the severity of depression: A higher score corresponds to more serious condition. On the basis of total score, the depressive symptoms can be divided into four grades: Severe depressive symptoms: total score greater than 35 points; moderate depressive symptoms: total score: 20-35 points; mild depressive symptoms: total score: $8-20$ points; and no depressive symptoms: total score less than 8 points.

\section{Research methods}

The total number of patients visited was 3,278, 317 of whom were diagnosed with depression, with 162 of them with abnormal cold and dry-type depression with comorbid anxiety.

A total of 150 patients with depression that was confirmed based on the abnormal cold and dry-type (sewda) syndrome diagnostic criteria were randomly divided into three groups (50 patients in each group): US therapy group, Fluoxetine therapy group, and US combined with Fluoxetine therapy group. Three weeks were regarded as the course of treatment. The test scores were evaluated at zero, three, and six weeks according to the HAMA, i.e., the US symptom score.

\section{Treatment method}

Patients belonging to Fluoxetine group were selected as the control group, and the treatment methods were as follows: (1) US treatment group: oral administration of $50 \mathrm{~mL}$ of US, twice a day. (2) US and Fluoxetine combined treatment group: oral administration of 20-mg Fluoxetine hydrochloride tablets, once in the morning; after $2 \mathrm{~h}$, oral administration of $50 \mathrm{~mL}$ of US. (3) Modern medicine control group (Fluoxetine treatment group): oral administration of 20-mg Fluoxetine tablets, once in the morning.

\section{Implementation of the survey}

Eligible participants were asked to fill in the details of the questionnaire. Simultaneously, the investigators must ensure that the questionnaire data fulfilled timeliness, completeness, accuracy, and clarity prior to the examination of the tongue and two hands (bilateral pulse).

\section{Quality control}

(1) To ensure the smooth progress of clinical research, investigators should develop and design reasonable clinical research program on the basis of previous literature reviews and experiences. (2) To ensure the reliability of the results, the patients must be grouped randomly in accordance with the random code packet envelope for avoiding partial selectivity, and then given different treatments. (3) To reduce the measurement bias in the evaluation process, the clinical cases 
observation table ought to be produced in accordance with the relevant standards of Good Clinical Practice (GCP). (4) To ensure the stability of drug quality and to improve the reliability of research conclusions, the acquisition of subject group must be accomplished in the process of drugs implementation. (5) To ensure the integrity of the clinical research data by telephone appointment, the ID number, address, and telephone number of each patient must be recorded in the study. (6) To ensure the accuracy of statistical data, the obtained clinical data must be verified before drawing any conclusions.

\section{Statistical analysis}

All clinical data collected in this research were entered into Excel database and analyzed by SPSS v21.0 statistical software. Measurement data including the mean and standard deviation $(\bar{x} \pm s)$ of the above-mentioned groups were analyzed using the Analysis of variance (ANOVA), and the twosample comparisons among the means were done by the LSD method. Chi-square test was used for comparison between groups of the counting data. Rank-sum test was used for comparison between groups of the ranking data. GraphPad Prism 5.0 was used for plotting. A $p<0.05$ was considered to be statistically significant.

\section{Results}

\section{Characteristic information of inclusion cases}

To assess the effect of US on the physical condition of patients with depression, 150 patients were randomly divided into three groups, then given oral administration of different medicines with Fluoxetine as the control (Fig. 1). Obviously, the results of HAMA $(F=0.745, p=0.481)$ exhibited no significance among the three groups (Table 1). Also, we explored several common factors that may influence the therapeutic activity of drugs, including age, sex, educational level, and marital status, then found that the $p$-values corresponding to these factors were all greater than 0.05 (Table 1), manifesting that a favorable curative effect of US on depression with comorbid anxiety cannot be prevented by external factors. Together, our data evidenced that US may be a novel Uyghur medicine for curing depression.

\section{Comparative analysis of therapeutic effects by Hamilton depression scale}

We can visually observe the variation trends of the total score response on a time scale from Table 2 and Figs. 2 and 3, which provided the results of HAMA for the three groups of patients at zero and six weeks after the treatment with US, Fluoxetine, or the combination of Fluoxetine and US.

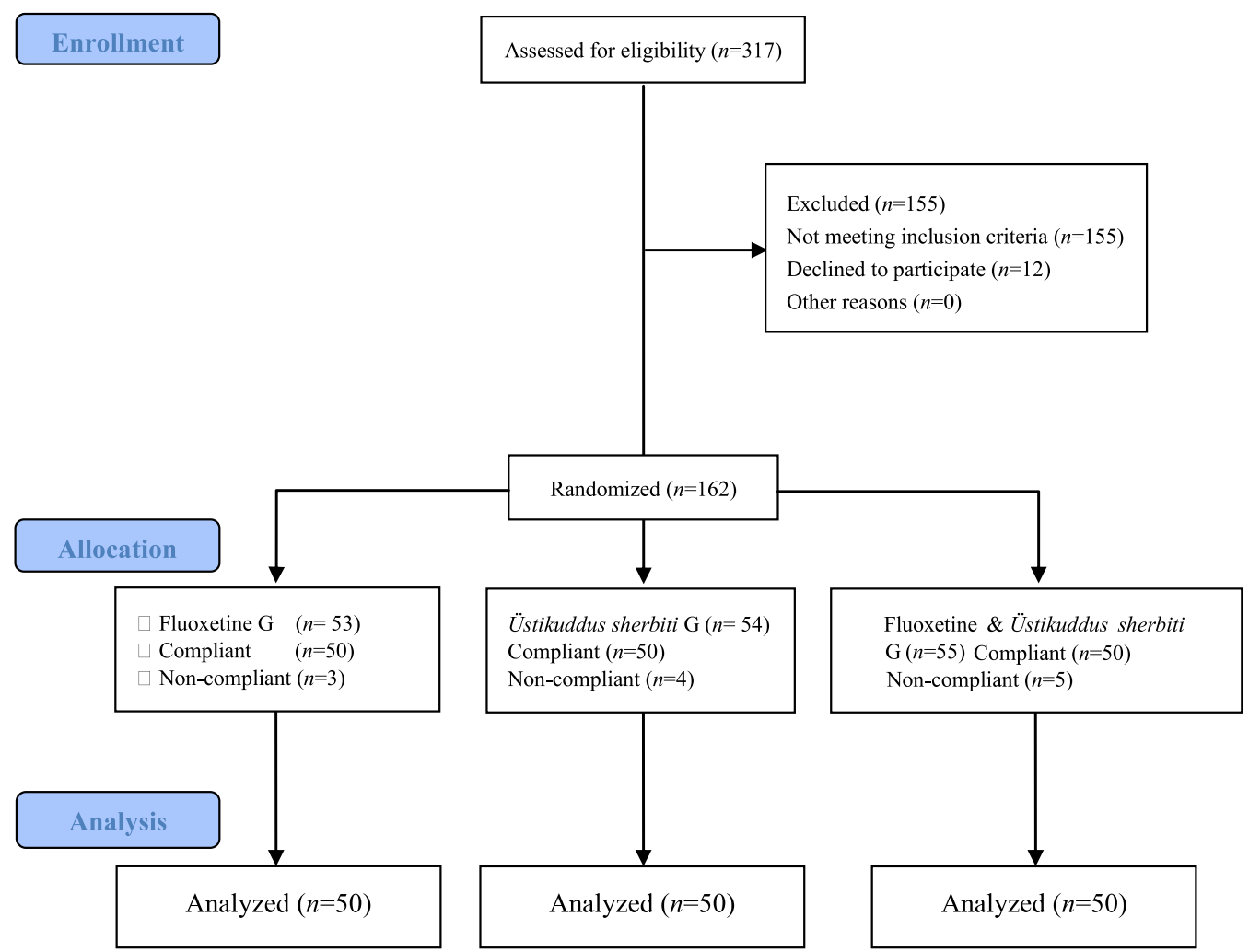

Figure 1. Overview of inclusion, division into three research groups, and the numbers of non-compliant and compliant patients. 
Table 1. Characteristics of the study samples (baseline patient characteristics).

\begin{tabular}{|c|c|c|c|c|c|}
\hline Group & Üstikuddus sherbiti $\mathrm{G}$ & Fluoxetine G & Combination $\mathrm{G}$ & $X^{2} / F$ & $p$-Value \\
\hline Age (years) & $48.80 \pm 13.66$ & $47.7 \pm 13.43$ & $45.66 \pm 12.30$ & 0.745 & 0.481 \\
\hline Sex & & & & 4.524 & 0.104 \\
\hline Male & $10(20.0)$ & $19(38.0)$ & $18(36.0)$ & & \\
\hline Female & $40(80.0)$ & $31(62.0)$ & $32(64.0)$ & & \\
\hline Education & & & & 3.381 & 0.184 \\
\hline Primary school & $2(4.0)$ & $2(4.0)$ & $5(10.0)$ & & \\
\hline Junior middle school & $5(10.0)$ & $9(18.0)$ & $12(24.0)$ & & \\
\hline High school & $12(24.0)$ & $15(30.0)$ & $9(18.0)$ & & \\
\hline Technical school & $5(10.0)$ & $2(4.0)$ & $1(2.0)$ & & \\
\hline Junior college & $6(12.0)$ & $5(10.0)$ & $9(18.0)$ & & \\
\hline Bachelor & $20(40.0)$ & $17(34.0)$ & $14(28.0)$ & & \\
\hline Marital status & & & & 7.618 & 0.079 \\
\hline Unmarried & $4(8.0)$ & $6(12.0)$ & $9(18.0)$ & & \\
\hline Married & $41(82.0)$ & $43(86.0)$ & $41(82.0)$ & & \\
\hline Other & $5(10.0)$ & $1(2.0)$ & $0(0.0)$ & & \\
\hline
\end{tabular}

Table 2. HAMA scores and levels before and after treatment for the three intervention groups.

\begin{tabular}{|c|c|c|c|c|c|c|}
\hline \multirow[b]{2}{*}{ Group } & \multicolumn{3}{|c|}{ Pretreatment } & \multicolumn{3}{|c|}{ Post-treatment } \\
\hline & $\begin{array}{c}\text { Üstikuddus } \\
\text { sherbiti G (50) }\end{array}$ & $\begin{array}{c}\text { Combination } \\
\text { G }(50)\end{array}$ & $\begin{array}{l}\text { Fluoxetine } \\
\text { G (50) }\end{array}$ & $\begin{array}{c}\text { Üstikuddus } \\
\text { sherbiti G (50) }\end{array}$ & $\begin{array}{l}\text { Combination } \\
\text { G }(50)\end{array}$ & $\begin{array}{l}\text { Fluoxetine } \\
\text { G (50) }\end{array}$ \\
\hline Somatic anxiety & $12.36 \pm 4.06$ & $12.22 \pm 4.15$ & $12.74 \pm 2.26$ & $8.50 \pm 3.76$ & $6.64 \pm 2.77$ & $8.98 \pm 2.50$ \\
\hline Cognitive anxiety & $13.78 \pm 3.55$ & $14.98 \pm 3.28$ & $15.16 \pm 1.94$ & $10.54 \pm 3.28$ & $9.56 \pm 2.86$ & $11.58 \pm 2.61$ \\
\hline Total score & $26.14 \pm 6.99$ & $27.20 \pm 6.63$ & $27.90 \pm 3.51$ & $19.02 \pm 5.69$ & $16.16 \pm 4.90$ & $20.56 \pm 4.35$ \\
\hline \multicolumn{7}{|l|}{ Diagnosis } \\
\hline Not present & $1(2.0)$ & $0(0.0)$ & $0(0.0)$ & $8(16.0)$ & $15(30.0)$ & $3(6.0)$ \\
\hline Mild or moderate & $7(14.0)$ & $6(12.0)$ & $1(2.0)$ & $23(46.0)$ & $30(60.0)$ & $24(48.0)$ \\
\hline Severe & $25(50.0)$ & $27(54.0)$ & $29(58.0)$ & $19(38.0)$ & $5(10.0)$ & $23(46.0)$ \\
\hline Very severe & $17(34.0)$ & $17(34.0)$ & $20(40.0)$ & $0(0.0)$ & $0(0.0)$ & $0(0.0)$ \\
\hline
\end{tabular}
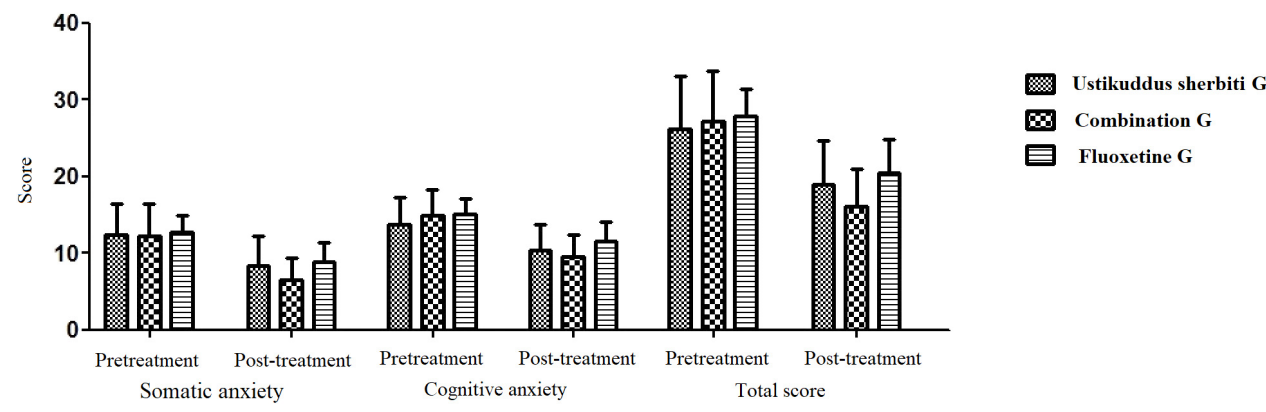

Figure 2. HAMA scores and levels before and after treatment for the three intervention groups.

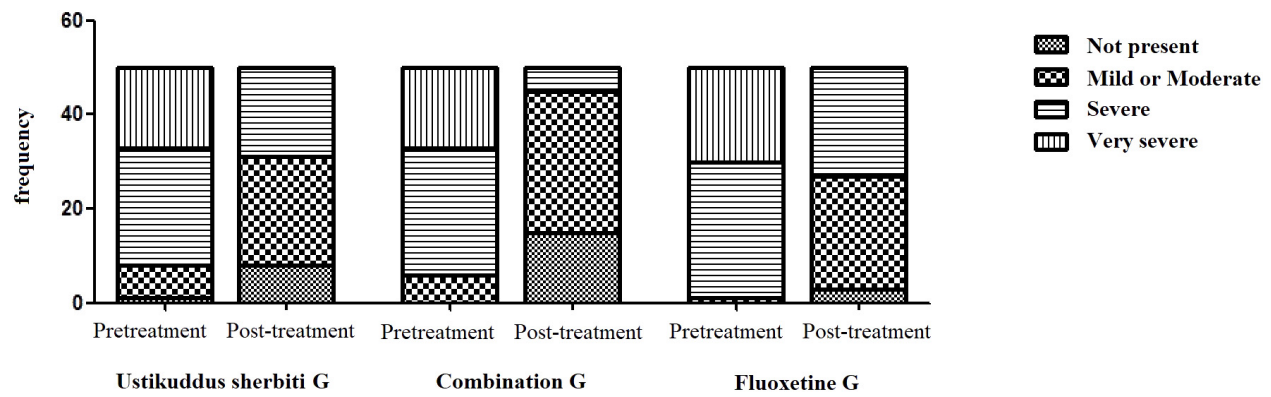

Figure 3. Analysis of outcomes from HAMA score reductions after treatment for the three intervention groups. 
Table 3. Analysis of outcomes from HAMA score reductions after treatment for the three intervention groups.

\begin{tabular}{lccc}
\hline & $\begin{array}{c}\text { Ustikuddus } \\
\text { sherbiti } \mathrm{G}(50)\end{array}$ & $\begin{array}{c}\text { Combination } \\
\mathrm{G}(50)\end{array}$ & $\begin{array}{c}\text { Fluoxetine } \\
\mathrm{G}(50)\end{array}$ \\
\hline Somatic anxiety & $3.86 \pm 3.6$ & $5.58 \pm 3.33$ & $3.76 \pm 2.33$ \\
Cognitive anxiety & $3.24 \pm 3.55$ & $5.42 \pm 3.44$ & $3.58 \pm 2.48$ \\
Total score & $7.12 \pm 5.14$ & $11.04 \pm 5.44$ & $7.34 \pm 3.43$ \\
\hline
\end{tabular}

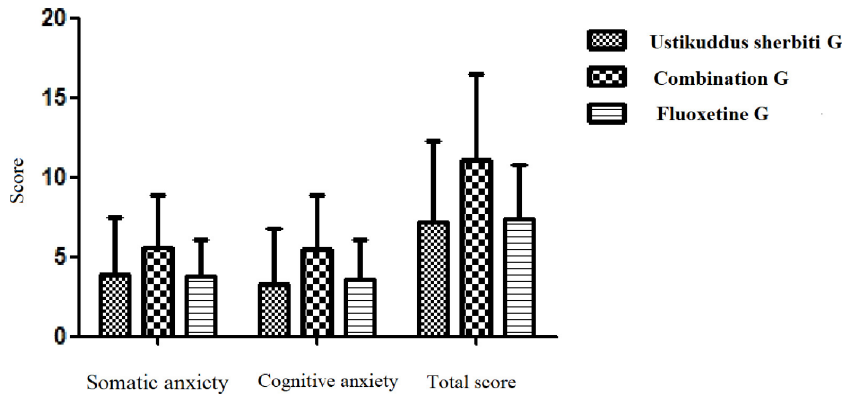

Figure 4. Comparisons of somatic anxiety and cognitive anxiety after treatment in the three intervention groups.

Clearly, the total score of HAMA for the three groups of patients gradually decreased with the passage of time; a more obvious decrease was observed in the combination group, indicating a better anti-depressant effect of the combination group compared with Fluoxetine or US group separately. The results of reduction of HAMA total scores for somatic anxiety and cognitive anxiety after six weeks of treatment were shown in Table 3 and Fig. 4. Obviously, the total score of HAMA for the three groups of patients gradually decreased till the sixth week. A more obvious decrease was observed in the combination group, also indicating a better anti-depressant effect of combination group compared with Fluoxetine or US alone.

\section{Adverse reaction}

During the six weeks of oral administration, we regularly detected various physical indicators of the patients. Unsurprisingly, there was no unpleasant symptoms in the physique and psychology observed during the whole course of medication, indicating US would not contribute to side effects for patients in the short term. Nevertheless, we will continue to observe patient's physical condition in a follow-up experiment for confirming the long-term effects of US.

\section{Discussion}

Typical symptoms of depression, a common mental illness in daily life, are exhibited as black mood, slow thinking, a reduction of language, and bradykinesia. ${ }^{1,2}$ Depression severely affects the life and work of patients and results in heavy burden to families and society, besides, about $15 \%$ of depression patients die because of suicide every year. Comorbid anxiety with depression predicts poor outcomes with a higher percentage of treatment resistance than either of the disorders occurring alone. The prevalence of comorbid anxiety disorder and MDD is frequent and perhaps as high as more than half. ${ }^{27,28}$ Overlap of anxiety and depression complicates the diagnosis and renders the treatment challenging.

Humorism of TUM (body fluid, liquid) and typical therapy of munziq (ripener, maturing agent) and mushil (scavenger, remover) exhibit significant advantages and characteristics in the prevention and treatment of complex diseases. ${ }^{19}$ Depression is called one of the abnormal cold and dry-type disorders of brain in TUM, which is the main reason of major diseases, such as MDD, cancer, dementia, and so on. These diseases mainly result from the damage of organ function induced by abnormal cold and dry-type body fluid. Especially, MDD has a common characteristic that is "the same disease with different syndromes". Abnormal cold and dry-type depression disorder is the main type of depression, which confirms the argument of depression resulted from abnormal cold and dry-type disorders. Besides, TUM considers that regular, moderate taking of munziq (US) by the patients with abnormal cold and dry-type disorders can effectively prevent the occurrence and development of depression. Numerous studies have reported the good curative effect of each herb of US in the treatment of depression, with others being anti-oxidant, anti-inflammatory, anti-tumor, and cardiovascular protection activities. ${ }^{27,29-31}$ In this study, we compared the therapeutic effect of three medications (US, Fluoxetine, and US combined with Fluoxetine) on the MDD patients with depression according to the total score of HAMA that is the main indicator of the severity of depression. Unsurprisingly, three medications all led to a positive effect on the status of patients. Also, we used ANOVA to verify whether there was significant difference among the three groups. Furthermore, the total score of combination group was remarkably less than single US and Fluoxetine groups, which provided a support that US has an important auxiliary therapeutic effect for Fluoxetine. In conclusion, our data provided a new, effective treatment schedule for depression.

\section{Conclusions}

The results of the study showed that there was a significant effect of US in treating depression with comorbid anxiety, and US and Fluoxetine can improve the clinical curative effect of depression with comorbid anxiety, which provides a new way of thinking for clarifying the US molecular mechanism for the treatment of depression and potential target. 


\section{Acknowledgments}

This study was supported by the Grant 2017-01-04-2 from Xinjiang Local Production New Drug Research \& Development Project for Traditional Chinese Medicine and Ethnic Medicine.

\section{Conflict of Interest}

The authors declare no competing interests.

\section{References}

1. Andrade L, Caraveoanduaga JJ, Berglund P, et al. The epidemiology of major depressive episodes: Results from the International Consortium of Psychiatric Epidemiology (ICPE) Surveys. Int J Methods Psychiatr Res 2003;12(1):3-21.

2. Kessler RC, Berglund P, Demler O, et al. The epidemiology of major depressive disorder: Results from the National Comorbidity Survey Replication (NCS-R). JAMA 2003;289(23):3095-105.

3. Murray CJ, Lopez AD. Alternative projections of mortality and disability by cause 1990-2020: Global burden of disease study. Lancet 1997;349(9064):1498-504.

4. Hasin DS, Goodwin RD, Stinson FS, Grant BF. Epidemiology of major depressive disorder: Results from the National Epidemiologic Survey on Alcoholism and Related Conditions. Arch Gen Psychiatry 2005;62:1097-106.

5. Farabaugh A, Bitran S, Nyer M, et al. Depression and suicidal ideation in college students. Psychopathology 2012;45(4):228-34.

6. Pinder-Amaker S, Auerbach RP, Rauch SL. Matching institutional needs with epidemiological demands: Addressing the mental health crisis across college campuses. Harv Health Policy Rev 2012;13:21-6.

7. Rab F, Mamdou R, Nasir S. Rates of depression and anxiety among female medical students in Pakistan. Eastern Mediterr Health J 2008;14(1):126-33.

8. Bongongo T. Screening for depression among adult patients on antiretroviral therapy for human immunodeficiency virus (HIV) attending primary health care facilities in the Rustenburg District. Thesis, University of Limpopo, 2010:1-52.

9. Nabeshima T, Kim HC. Involvement of genetic and environmental factors in the onset of depression. Exp Neurobiol 2013;22:235-43.

10. Hou Z, Jiang W, Yin Y, et al. The current situation on major depressive disorder in China: Research on mechanisms and clinical practice. Neurosci Bull 2016;32:389-97.

11. Huppert JD, Simpson HB, Nissenson KJ, et al. Quality of life and functional impairment in obsessive-compulsive disorder: A comparison of patients with and without comorbidity, patients in remission, and healthy controls. Depress Anxiety 2009;26(1):39-45.

12. Goldstein-Piekarski AN, Williams LM, Humphreys K. A transdiagnostic review of anxiety disorder comorbidity and the impact of multiple exclusion criteria on studying clinical outcomes in anxiety disorders. Transl Psychiatry 2016;6(6):e847.

13. Wu Z, Fang Y. Comorbidity of depressive and anxiety disorders: Challenges in diagnosis and assessment. Shanghai Arch Psychiatry 2014;26(4):227-31.
14. Santarsieri D, Schwartz TL. Antidepressant efficacy and sideeffect burden: A quick guide for clinicians. Drugs in Context 2015;4:1-12.

15. Leventhal AM. Sadness, depression, and avoidance behavior. Behav Modif 2008;32(6):759-79.

16. Timonen M, Liukkonen T. Management of depression in adults. BMJ 2008;336(7641):435-9.

17. Qiu X, Yang X, Qiao Z, et al. Impairment in processing visual information at the pre-attentive stage in patients with a major depressive disorder: A visual mismatch negativity study. Neuroscience Lett 2011;491:53-7.

18. Adli M, Pilhatsch M, Bauer M, et al. Safety of high-intensity treatment with the irreversible monoamine oxidase inhibitor tranylcypromine in patients with treatment-resistant depression. Pharmacopsychiatry 2008;41:252-7.

19. Dong JC. Comparative Study of Traditional Chinese Medicine. Shanghai: Shanghai Science and Technology Press, 2019, pp. 60, 262-271, 328-336, 391-398, 542-543, 601-604.

20. Gul A, Wushuer P, Rui H, et al. A survey and analysis of traditional Uyghur medicine abnormal body fluid type major depressive disorder with personality traits. Tradit Med Mod Med 2019;02(01):13-8.

21. Moore N, Hamza N, Berke B, Umar A. News from Tartary: An ethnopharmacological approach to drug and therapeutic discovery. Br J Clin Pharmacol 2017;83(1):33-7.

22. Yishakejiang M, Abudureyimu K, Abulake R. China Medical Encyclopedia - Uyghur Medicine Volume. Shanghai: Shanghai Science and Technology Press, 2005.

23. Amiti A, Abudukadi J, Wufuer R. Clinical study of Uygur medicine adjusting temperament method combined with Üstikuddus sherbiti (Yinao Wustikudusi Tangjiang) in treating female climacteric depression. J Med Pharm Chin Minorities 2017;6:1-3.

24. Wufuer R, Amiti A. Effect of munziq sewda mixture combined with modern medicine on treatment of HIV/AIDS patients with pelvic abscess. Chin J Mod Drug Appl 2016;10:172-3.

25. Nurmuhammat M, Musa H, Axim T, Talip A. Retrospective investigation on hospitalized records of Uygur medical treatment for insomnia. Chin J Inf Tradit Chin Med 2013;28:16-8.

26. Vilalta-Franch J, Garre-Olmo J, López-Pousa S, et al. Comparison of different clinical diagnostic criteria for depression in Alzheimer disease. Am J Geriatr Psychiatry 2006;14:589-97.

27. Soheili M, Tavirani M, Salami M. Lavandula angustifolia extract improves deteriorated synaptic plasticity in an animal model of Alzheimer's disease. Iran J Basic Med Sci 2015;18 (11):1147-52.

28. Anushiravani M, Manteghi AA, Taghipur A, Eslami M. Depression from the perspective of modern and Persian medicine. Electron Physician 2018;10:6372-8.

29. Rahman M, Sahabjada S, Akhtar J. Evaluation of anticancer activity of Cordia dichotoma leaves against a human prostate carcinoma cell line, PC3. J Tradit Complement Med 2016;38:121-6.

30. Sun Z, Park S, Hwang E, et al. Dietary Foeniculum vulgare Mill extract attenuated UVB irradiation-induced skin photoaging by activating of Nrf2 and inhibiting MAPK pathways. Phytomedicine 2016;5:1273-84.

31. Xu P, Wang K, Lu C, et al. Protective effect of lavender oil on scopolamine induced cognitive deficits in mice and $\mathrm{H}_{2} \mathrm{O}_{2}$ induced cytotoxicity in PC12 cells. J Ethnopharmacol 2016; 193:408-15. 